\title{
Adaptive Inventory Control Based on Fuzzy Neural Network under Uncertain Environment
}

\author{
Jianqiao $\mathrm{Ge}^{1}$ and Songtao Zhang $\mathbb{D}^{2}$ \\ ${ }^{1}$ School of Management, Harbin University of Commerce, Harbin 150028, China \\ ${ }^{2}$ School of Logistics, Linyi University, Linyi 276005, China \\ Correspondence should be addressed to Songtao Zhang; zst0626@163.com
}

Received 14 May 2020; Accepted 13 July 2020; Published 29 July 2020

Academic Editor: Qingling Wang

Copyright (C) 2020 Jianqiao Ge and Songtao Zhang. This is an open access article distributed under the Creative Commons Attribution License, which permits unrestricted use, distribution, and reproduction in any medium, provided the original work is properly cited.

\begin{abstract}
In order to achieve the actual inventory effectively tracking the target inventory under uncertain environment, this paper investigates an adaptive inventory controller for the production-inventory system. First, an uncertain production-inventory model is constructed, and then, the uncertainty of the production-inventory model is approximated by a fuzzy neural network. Secondly, in terms of the design of adaptive control law, the adaptive inventory controller is developed. Under the adaptive inventory controller, the actual inventory can track the target inventory in real time and the production-inventory system can be robustly stable in uncertain environment. Finally, the results of three simulation experiments show that the proposed adaptive inventory controller can realize both the fast tracking speed and the high tracking accuracy.
\end{abstract}

\section{Introduction}

Inventory management is always the core problem of enterprises. Sufficient inventory can improve the response speed of enterprises to the market, reduce the risk of shortage, and shorten the delivery time of products. However, on the one hand, excessive inventory will occupy a large amount of working capital and increase the inventory cost; on the other hand, too little inventory will deprive enterprises of sales opportunities.

In the last few decades, scholars have made a systematic study on inventory management. In traditional inventory management, the representative models are the EOQ model [1-6] and the $(s, S)$ model [7-9]. For example, Tavakoli and Taleizadeh [2] constructed an EOQ model for decaying item with conditional discount and full advanced payment; Chiarini [5] modified the EOQ model to match the just-intime quasi-pull production environment; Castellano et al. [7] dealt with the application of a Bernoulli model to the case of a single-vendor single-buyer supply chain with $(s, S)$ inventory policy; considering a finite-horizon single-product periodic-review inventory management problem with demand distribution uncertainty, Qiu et al. [9] proved the existence of an optimal $(s, S)$ policy.

In addition to the classic research of inventory management, some scholars applied the control theory to inventory management and formed a unique inventory control theory. In this field, there is a family of APIOBPCS (Automatic Pipeline Inventory with Order-Based Production Control System) models [10-16], whose principle is to transform the differential equation into the transfer function of the control system by the Laplace transform and to add elements such as automatic pipeline. For example, Hosseini and Mehrjerdi [12] investigated the impact of different inventory policies on the APIOBPCS model; Zhan et al. [16] built up a model of VMI (Vendor Managed Inventory)APIOBPCS for the apparel supply chain. Besides IOBPCS family, there are other control methods applied to inventory management, for example, Subramanian et al. [17] proposed the economic MPC (model predictive control) to guarantee the closed-loop properties for supply chain optimization.

However, the inventory level is often influenced by some random events, such as inaccurate prediction, mechanical failure, strikes, and other internal events or external 
disturbances, and then, the inventory system under the classical control methods may take place a significant decline in performance, or even instability.

Because the adaptive control method is an effective method to suppress the impact of random events on the system [18-28], some scholars applied the adaptive control method to inventory control to avoid the defects of the classical control methods in inventory control, for example, Aggelogiannaki et al. [20] proposed an adaptive MPC configuration to identify and control the production-inventory systems. Foul et al. [21] introduced the model reference adaptive control with feedback to track the inventory level. Applying online adaptation in the framework of passivity-based control, Li et al. [22] developed an adaptive inventory control strategy. Mandel [23] presented a set of models and algorithms for adaptive inventory control in the case of uncertainty. Aengchuan and Phruksaphanrat [24] compared the inventory control effects of the fuzzy inference system model, artificial neural network model, and adaptive neural fuzzy inference system model. Azarskov et al. [25] proposed an adaptive control method to improve the performance of the inventory control system under uncertainty. Based on Kalman filtering, Vladimir and Kenne [26] developed an adaptive control method for the manufacturing systems with incomplete information about demand and inventory. Paul et al. [27] applied the adaptive neural fuzzy inference system to modeling the inventory problem. Fu et al. [28] quantified and mitigated the bullwhip effect in a benchmark supply chain system by an extended prediction self-adaptive control ordering policy.

In the literature mentioned above, only authors in $[9,18,23]$ and $[25]$ investigated the problem of inventory management under uncertainty, and only authors in [9] considered the robustness of inventory system, none of the authors paid enough attention to parameters adjusted online, and all authors for the adaptive control only set the target inventory as a constant. Under uncertain environment, only the robust production-inventory system can maintain its stable operation. Furthermore, for the variable target inventory, the number of parameters that can be adjusted online in the adaptive control algorithm directly reflects the tracking speed and the tracking accuracy in inventory control. Therefore, in our paper, for the periodic change of the target inventory, an adaptive inventory controller is proposed under uncertain environment caused by prediction error, machine failure, workers' strike, and so on. By three parameters adjusted online simultaneously, an adaptive control algorithm is designed so that the production-inventory system can track the target inventory with high accuracy and high speed. In addition, the robust controller is used to suppress the impact of uncertainties on the production-inventory system.

The rest of the paper is organized as follows: Section 2 constructs an uncertain production-inventory model. The uncertainty of the production-inventory model is approximated by the fuzzy neural network in Section 3. Section 4 develops an adaptive inventory controller. The simulation analysis is given in Section 5. Some conclusions are drawn in Section 6.

\section{Uncertain Production-Inventory Model}

The meanings of relevant symbols in the paper are shown in Table 1.

The production-inventory model under uncertain customers' demand is constructed in Figure 1.

In this model, $D(t)$ and $I(t)$ are the input variable and the output variable of the system, respectively. The prediction result $P(t)$ of $D(t)$ can be obtained from the following single exponential smoothing:

$$
G_{A}(S)=\frac{1}{T_{a} s+1} .
$$

In terms of the inventory deviation adjustment time $T_{I}$, the production plan is corrected to make the actual inventory level consistent with the target inventory level.

The transfer function of the production-inventory model can be obtained as follows:

$$
\begin{aligned}
F(S) & =\frac{I(s)}{D(s)}=\frac{\left(1 /\left(T_{a} s+1\right)\right) \cdot(1 / s)}{1+\left(1 / T_{I}\right) \cdot(1 / s)}-\frac{(1 / s)}{1+\left(1 / T_{I}\right) \cdot(1 / s)} \\
& =\frac{T_{I}}{\left(T_{a} s+1\right)\left(T_{I} s+1\right)}-\frac{T_{I}}{\left(T_{I} s+1\right)}=\frac{-T_{I} T_{a} s}{\left(T_{a} s+1\right)\left(T_{I} s+1\right)} .
\end{aligned}
$$

The differential equation of equation (2) can be expressed as follows:

$$
\ddot{I}=-\dot{D}-\left(\frac{1}{T_{a}}+\frac{1}{T_{I}}\right) \dot{I}-\frac{1}{T_{a} T_{I}} I=f+u,
$$

where $f=-\left(\left(1 / T_{a}\right)+\left(1 / T_{I}\right)\right) \dot{I}-\left(1 / T_{a} T_{I}\right) I$ and $u=-\dot{D}$.

By considering the uncertain inventory deviation adjustment time caused by prediction error, commodity price fluctuation, machine failure, and workers' strike, and the uncertain consumption averaging time caused by the customers' demand in the production-inventory system, the uncertain production-inventory model is described as follows:

$$
\begin{aligned}
\ddot{I}= & -\dot{D}-\left(\frac{1}{T_{a}+\Delta T_{a}}+\frac{1}{T_{I}+\Delta T_{I}}\right) \dot{I} \\
& -\frac{1}{\left(T_{a}+\Delta T_{a}\right)\left(T_{I}+\Delta T_{I}\right)} I+\gamma,
\end{aligned}
$$

where $\Delta T_{a}$ and $\Delta T_{I}$ are the uncertain terms of the system parameters $T_{a}$ and $T_{I}$, respectively, and $\gamma$ denotes the uncertain term of the external disturbance. Equation (4) can be written as follows:

$$
\ddot{I}=f+f_{\Delta}+u+\gamma=f+u+\Omega,
$$

where $f_{\Delta}=\left(\left(\Delta T_{a} /\left(T_{a}\left(T_{a}+\Delta T_{a}\right)\right)\right)+\left(\Delta T_{I} /\left(T_{I}\left(T_{I}+\right.\right.\right.\right.$ $\left.\left.\left.\left.\Delta T_{I}\right)\right)\right)\right) \dot{I}+\left(\left(T_{a} \Delta T_{I}+T_{I} \Delta T_{a}+\Delta T_{a} \Delta T_{I}\right) /\left(T_{a} T_{I}\left(T_{a}+\Delta T_{a}\right)\right.\right.$ $\left.\left.\left(T_{I}+\Delta T_{I}\right)\right)\right) I$ and $\Omega=f_{\Delta}+\gamma$ is referred to lumped uncertainties.

Compared with the classic control methods, the adaptive control method can suppress the influence of disturbance on the production-inventory system and make the actual 
TABLE 1: Meanings of relevant symbols.

\begin{tabular}{lc}
\hline$\alpha$ & Stabilization function \\
$c_{j}$ & Matrix vector of the center \\
$\sigma_{j}$ & Matrix vector of the radius \\
$\pi_{j}$ & Connection weight between the hidden layer \\
$D$ & and the output layer \\
$e_{1}$ & Customers' demand \\
$e_{2}$ & Tracking error \\
$\varepsilon$ & Stabilization error \\
$F_{i}^{j}$ & Matching error \\
$G^{j}$ & Fuzzy set of membership function $\mu_{F_{i}^{j}}\left(x_{i}\right)$ \\
$I$ & Fuzzy set of membership function $\mu_{G^{j}}(y)$ \\
$I_{c}$ & Actual inventory level \\
$P$ & Target inventory level \\
$R$ & Prediction result of customers' demand \\
$T_{a}$ & Fuzzy rule \\
$T_{I}$ & Consumption averaging time \\
$\tau_{1}$ & Inventory deviation adjustment time \\
$\tau_{2}$ & A positive number \\
$U_{a}$ & Another positive number \\
$U_{b}$ & Basic fuzzy controller \\
$U_{a b}$ & Robust controller \\
$\Omega$ & Adaptive fuzzy controller \\
$\mu_{F_{i}^{j}}\left(x_{i}\right)$ & Error \\
$\mu_{G}(y)$ & Gaussian function \\
$V$ & Single point set function \\
$y$ & Lyapunov function \\
\hline &
\end{tabular}

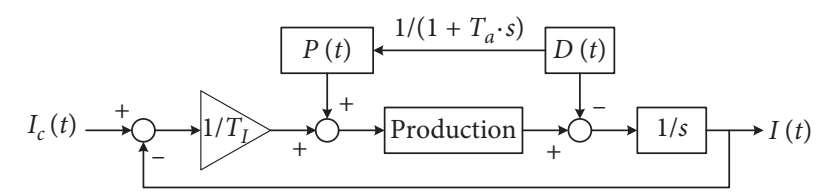

FIGURE 1: Production-inventory model.

inventory track the target inventory. Therefore, this paper will design an adaptive inventory controller to improve the tracking accuracy and the tracking speed.

\section{Expression of Uncertainty}

Before the adaptive inventory controller is designed, the uncertainty $\Omega$ in the production-inventory model will be approximated by the fuzzy neural network. Figure 2 shows the structure of the fuzzy neural network.

The function of each layer in the fuzzy neural network is described as follows:

Layer 1 (input layer): this layer defines input language variables: $x=\left[x_{1} x_{2} \cdots x_{n}\right]^{T} \in R^{n}$.

Layer 2 (fuzziness layer): each node in this layer represents a membership function. In our paper, Gaussian function is chosen as the membership function.

Layer 3 (rule layer): each node of this layer corresponds to a fuzzy rule.

Layer 4 (output layer): the node of this layer corresponds to the output variable.

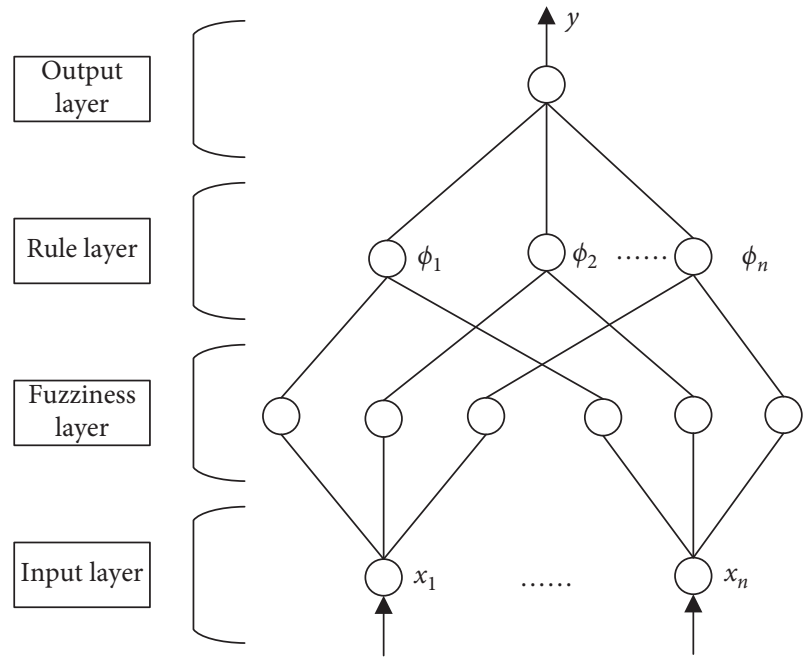

Figure 2: The structure of the fuzzy neural network.

The output of the fuzzy neural network is described as follows:

$$
y=\sum_{j=1}^{N} \pi_{j} \phi_{j}\left(\sigma_{j},\left\|I-c_{j}\right\|\right)=\pi^{T} \phi(I, c, \sigma),
$$

where $\quad \phi_{j}\left(\sigma_{j},\left\|I-c_{j}\right\|\right)=\prod_{i=1}^{n} \exp \left[-\left(I_{j}-c_{j}^{i}\right)^{2} / \sigma_{j}^{i 2}\right], \quad c_{j}=$ $\left[c_{j}^{1} c_{j}^{2} \cdots c_{j}^{n}\right]^{T} \in R^{n}, \quad \sigma_{j}=\left[\sigma_{j}^{1} \sigma_{j}^{2} \cdots \sigma_{j}^{n}\right]^{T} \in R^{n}, \quad \pi=\left[\pi_{1} \pi_{2} \cdots\right.$ $\left.\pi_{n}\right]^{T} \in R^{n}, \quad c=\left[c_{1}^{T} c_{2}^{T} \cdots c_{N}^{T}\right]^{T} \in R^{n N}$, and $\sigma=\left[\sigma_{1}^{T} \sigma_{2}^{T} \cdots\right.$ $\left.\sigma_{N}^{T}\right]^{T} \in R^{n N}$.

Equation (6) will approximate the lumped uncertainties $\Omega$ in equation (5). If the estimated value of $\Omega$ is $\widehat{\Omega}$, according to the universal approximation theory [29], there is an optimal approximation value $\Omega^{*}$, which satisfies the following equation:

$$
\Omega=\Omega^{*}\left(I, c^{*}, \sigma^{*}, \pi^{*}\right)+\Delta=\pi^{* T} \phi^{*}\left(I, c^{*}, \sigma^{*}\right)+\Gamma,
$$

where $\Gamma$ represents the approximation error. In [30], we assume $|\Gamma| \leq \Gamma^{*}, \Gamma^{*}$ is a positive constant. $c^{*}, \sigma^{*}$, and $\pi^{*}$ are the optimal parameter vectors of $c, \sigma$, and $\pi$, respectively. There exist constants $\bar{c}, \bar{\sigma}$, and $\bar{\pi}$ satisfying $\left\|c^{*}\right\| \leq \bar{c},\left\|\sigma^{*}\right\| \leq \bar{\sigma}$, and $\left\|\pi^{*}\right\| \leq \bar{\pi}$, respectively. The estimation function $\Omega$ can be defined as follows:

$$
\widehat{\Omega}(I, \widehat{c}, \widehat{\sigma}, \widehat{\pi})=\hat{\pi}^{T} \widehat{\phi}(I, \widehat{c}, \widehat{\sigma}) .
$$

In equation (8), $\bar{\phi}, \hat{\pi}, \bar{c}$, and $\widehat{\sigma}$ are the estimation vectors of $\phi^{*}, \pi^{*}, c^{*}$, and $\sigma^{*}$, respectively. For convenience, let $\phi^{*}=$ $\phi^{*}\left(I, c^{*}, \sigma^{*}\right)$ and $\widehat{\phi}=\widehat{\phi}(I, \widehat{c}, \widehat{\sigma})$.

The error $\widetilde{\Omega}$ is defined as follows:

$$
\widetilde{\Omega}=\Omega-\widehat{\Omega}=\Omega^{*}-\widehat{\Omega}+\Delta=\tilde{\pi}^{T} \widehat{\phi}+\tilde{\pi}^{T} \widetilde{\phi}+\tilde{\pi}^{T} \widetilde{\phi}+\Gamma,
$$

where $\tilde{\pi}=\pi^{*}-\tilde{\pi}$ and $\tilde{\phi}=\phi^{*}-\widehat{\phi}$.

In order to transform the nonlinear membership function equation into partial linearization form, using the two-place Taylor's formula, we can get

$$
\phi^{*}=\bar{\phi}+\left.\phi_{c}^{\prime}\right|_{c=\bar{c}} \tilde{c}+\left.\phi_{\sigma}^{\prime}\right|_{\sigma=\widetilde{\sigma}} \tilde{\sigma}+g(I, \widetilde{c}, \widetilde{\sigma}),
$$


where $\widetilde{c}=c^{*}-\widehat{c}, \widetilde{\sigma}=\sigma^{*}-\widehat{\sigma}$, and $g(I, \widetilde{c}, \widetilde{\sigma})$ is the sum of high order terms of Taylor series. $\widetilde{\phi}=\phi^{*}-\bar{\phi}$ can be expressed as follows:

$$
\widetilde{\phi}=\phi^{*}-\bar{\phi}=\left.\phi_{c}^{\prime}\right|_{c=\bar{c}} \tilde{c}+\left.\phi_{\sigma=\bar{\sigma}}^{\prime}\right|_{\sigma=} \tilde{\sigma}+g(I, \tilde{c}, \widetilde{\sigma}) .
$$

$g(I, \widetilde{c}, \widetilde{\sigma}), \widetilde{c}, \widetilde{\sigma}$, and $\widetilde{\pi}$ are bounded; then

$$
\begin{aligned}
\|g(I, \widetilde{c}, \widetilde{\sigma})\| & =\left\|\widetilde{\phi}-\phi_{c}^{\prime} \widetilde{c}-\phi_{\sigma}^{\prime} \widetilde{\sigma}\right\| \leq\|\widetilde{\phi}\|+\left\|\phi_{c}^{\prime}\right\|\|\widetilde{c}\|+\left\|\phi_{\sigma}^{\prime}\right\|\|\widetilde{\sigma}\| \\
& \leq k_{1}+k_{2}\|\widetilde{c}\|+k_{3}\|\widetilde{\sigma}\|,
\end{aligned}
$$

where $\|\widetilde{\phi}\| \leq k_{1},\left\|\phi_{c}^{\prime}\right\| \leq k_{2},\left\|\phi_{\sigma}^{\prime}\right\| \leq k_{3}, k_{1}, k_{2}$, and $k_{3}$ are all bounded positive numbers, $\|\widetilde{c}\|=\left\|c^{*}-\bar{c}\right\| \leq\left\|c^{*}\right\|+$ $\|\bar{c}\| \leq \bar{c}+\|\bar{c}\|, \quad\|\widetilde{\sigma}\|=\left\|\sigma^{*}-\bar{\sigma}\right\| \leq\left\|\sigma^{*}\right\|+\|\bar{\sigma}\| \leq \bar{\sigma}+\|\widehat{\sigma}\|, \quad$ and $\|\tilde{\pi}\|=\left\|\pi^{*}-\widehat{\pi}\right\| \leq\left\|\pi^{*}\right\|+\|\bar{\pi}\| \leq \bar{\pi}+\|\bar{\pi}\|$.

Substituting equation (11) into equation (9), we have

$$
\begin{aligned}
\widetilde{\Omega} & =\tilde{\pi}^{T} \hat{\phi}+\tilde{\pi}^{T}\left(\phi_{c}^{\prime} \widetilde{c}+\phi_{\sigma}^{\prime} \widetilde{\sigma}+g\right)+\tilde{\pi}^{T} \tilde{\phi}+\Delta \\
& =\tilde{\pi}^{T} \hat{\phi}+\tilde{c}^{T} \phi_{c}^{T} \tilde{\pi}+\tilde{\sigma}^{T} \phi_{\sigma}^{T} \tilde{\pi}+\tilde{\pi}^{T} g+\tilde{\pi}^{T} \tilde{\phi}+\Delta \\
& =\tilde{\pi}^{T} \hat{\phi}+\tilde{c}^{T} \phi_{c}^{T} \widetilde{\pi}+\widetilde{\sigma}^{T} \phi_{\sigma}^{T} \widetilde{\pi}+\varepsilon .
\end{aligned}
$$

For the scalar matrices in equation (13), we have $\tilde{c}^{T} \phi_{c}^{\prime} T \widehat{\pi}=\bar{\pi}^{T} \phi_{c}^{\prime} \widetilde{c}$ and $\widetilde{\sigma}^{T} \phi_{\sigma}^{\prime T} \tilde{\pi}=\tilde{\pi}^{T} \phi_{\sigma}^{\prime} \widetilde{\sigma}$. The matching error $\varepsilon=\tilde{\pi}^{T} g+\tilde{\pi}^{T} \tilde{\phi}+\Gamma$, and $\|\varepsilon\| \leq E$, where $E$ is a positive number. It is difficult to determine the exact value of $E$ in practical application.

\section{Design of Adaptive Inventory Controller}

The adaptive inventory controller designed in this paper consists of a fuzzy neural network controller $u_{a}(t)$ and a robust controller $u_{b}(t)$. In the adaptive inventory controller, the fuzzy neural network is used to deal with the problem of uncertainties, the feedback control is used to track the target inventory, an adaptive algorithm is used to solve the problem of automatic online adjustment of parameters, and the robust controller is used to improve the robustness of the production-inventory system.

When the inventory control is carried out, the error between the actual inventory and the target inventory is inevitable, which can be expressed as follows:

$$
I_{c}(t)=I(t)-e_{1}(t) .
$$

Taking the derivative of equation (14), we have

$$
\dot{I}_{c}(t)=\dot{I}(t)-\dot{e}_{1}(t) \text {. }
$$

As a virtual control variable, $\dot{I}(t)$ can be represented as the following form based on the stabilizing function $a(t)$ :

$$
\dot{I}_{c}(t)=\tau_{1} e_{1}(t)+a(t)
$$

For the steady-state error, we have

$$
e_{2}(t)=\dot{I}(t)-a(t)=\dot{e}_{1}(t)+\tau_{1} e_{1}(t) .
$$

Then, the derivative of the steady-state error can be expressed as follows:

$$
\begin{aligned}
\dot{e}_{2}(t) & =\ddot{I}(t)-\dot{a}(t)=\ddot{I}(t)-\left[-\tau_{1} \dot{e}_{1}(t)+\ddot{I}_{c}(t)\right] \\
& =f+u+\widehat{\Omega}+\tau_{1} e_{2}(t)-\tau_{1}^{2} e_{1}(t)-\ddot{I}_{c}(t) .
\end{aligned}
$$

The adaptive inventory controller is designed as follows:

$$
\begin{aligned}
u(t) & =u_{a}(t)+u_{b}(t), \\
u_{a}(t) & =\ddot{I}_{c}(t)-f-\widehat{\Omega}+\left(\tau_{1}^{2}-1\right) e_{1}(t)-\left(\tau_{1}+\tau_{2}\right) e_{2}(t),
\end{aligned}
$$

$$
u_{b}(t)=-\hat{E} \operatorname{sgn}\left(e_{2}(t)\right),
$$

where $\widehat{E}$ is the estimate of $E$.

In order to determine the adaptive law and the estimation algorithm of boundary value in the adaptive inventory controller, substituting equation (19), equation (20), and (21) into (5), we have

$$
\dot{e}_{2}(t)=\Omega-\widehat{\Omega}-\tau_{2} e_{2}(t)-e_{1}(t)-\widehat{E} \operatorname{sgn}\left(e_{2}(t)\right) .
$$

Substituting equation (13) into equation (22), we have

$$
\begin{aligned}
\dot{e}_{2}(t)= & \tilde{\pi}^{T} \hat{\phi}+\widetilde{c}^{T} \phi_{c}^{T} \hat{\pi}+\widetilde{\sigma}^{T} \phi_{\sigma}^{\prime T} \hat{\pi}+\varepsilon-\tau_{2} e_{2}(t) \\
& -e_{1}(t)-\widehat{E} \operatorname{sgn}\left(e_{2}(t)\right) .
\end{aligned}
$$

According to the idea in [31], we propose the following theorem.

Theorem 1. The estimation algorithm of boundary value and the adaptive law of parameters are given as follows:

$$
\begin{aligned}
& \ddot{\widetilde{E}}(t)=-\dot{\widetilde{E}}(t)=\eta_{1}\left|e_{2}(t)\right| \text {, } \\
& \ddot{\bar{\pi}}=-\dot{\tilde{\pi}}=\eta_{2} e_{2}(t) \hat{\phi} \text {, } \\
& \ddot{\vec{c}}=-\dot{\tilde{c}}=\eta_{3} e_{2}(t) \phi_{\bar{c}}^{\prime} \hat{\pi} \text {, } \\
& \ddot{\widetilde{\sigma}}=-\dot{\tilde{\sigma}}=\eta_{4} e_{2}(t) \phi_{\tilde{\sigma}}^{\prime} T \text {. }
\end{aligned}
$$

Then $\Gamma$ is bounded and $\|\varepsilon\| \leq E$, and then $\lim \left[I(t)-I_{c}(t)\right]=0$, where $\widetilde{E}(t)=E-\widehat{E}(t)$, and $\eta_{1}, \eta_{2}$, $\underset{\eta_{3}}{\longrightarrow}$, and $\eta_{4}$ are all positive.

Proof. A Lyapunov function is defined as follows:

$$
\begin{aligned}
V\left(e_{1}(t), e_{2}(t), \widetilde{E}(t), \tilde{\pi}, \widetilde{c}, \widetilde{\sigma}\right)= & \frac{e_{1}^{2}(t)}{2}+\frac{e_{2}^{2}(t)}{2}+\frac{\widetilde{E}^{2}(t)}{2 \eta_{1}}+\frac{\widetilde{\pi}^{T} \widetilde{\pi}}{2 \eta_{2}} \\
& +\frac{\widetilde{c}^{T} \widetilde{c}}{2 \eta_{3}}+\frac{\widetilde{\sigma}^{T} \widetilde{\sigma}}{2 \eta_{4}}=V_{1}(t)+V_{2}(t),
\end{aligned}
$$

where $\quad V_{1}(t)=\left(e_{1}^{2}(t) / 2\right)+\left(e_{2}^{2}(t) / 2\right) \quad$ and $V_{2}(t)=\left(\widetilde{E}^{2}(t) / 2 \eta_{1}\right)+\left(\tilde{\pi}^{T} \widetilde{\pi} / 2 \eta_{2}\right)+\left(\widetilde{c}^{2} \widetilde{c} / 2 \eta_{3}\right)+\left(\widetilde{\sigma}^{T} \widetilde{\sigma} / 2 \eta_{4}\right)$. Based on equation (23), the derivative of the Lyapunov function can be expressed as follows: 

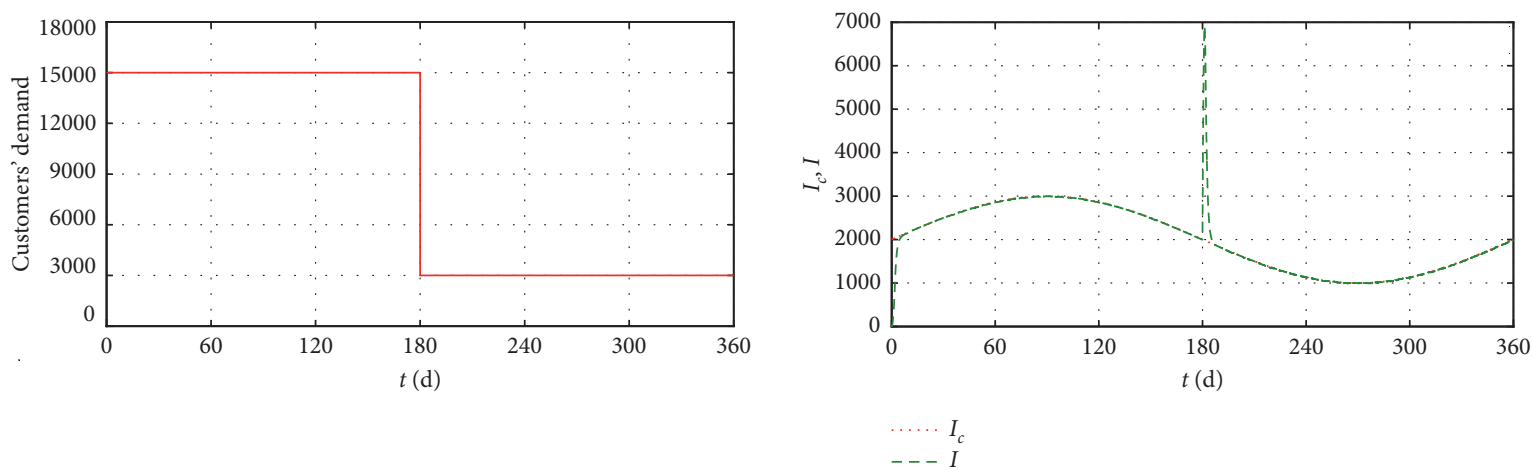

(a)

(b)
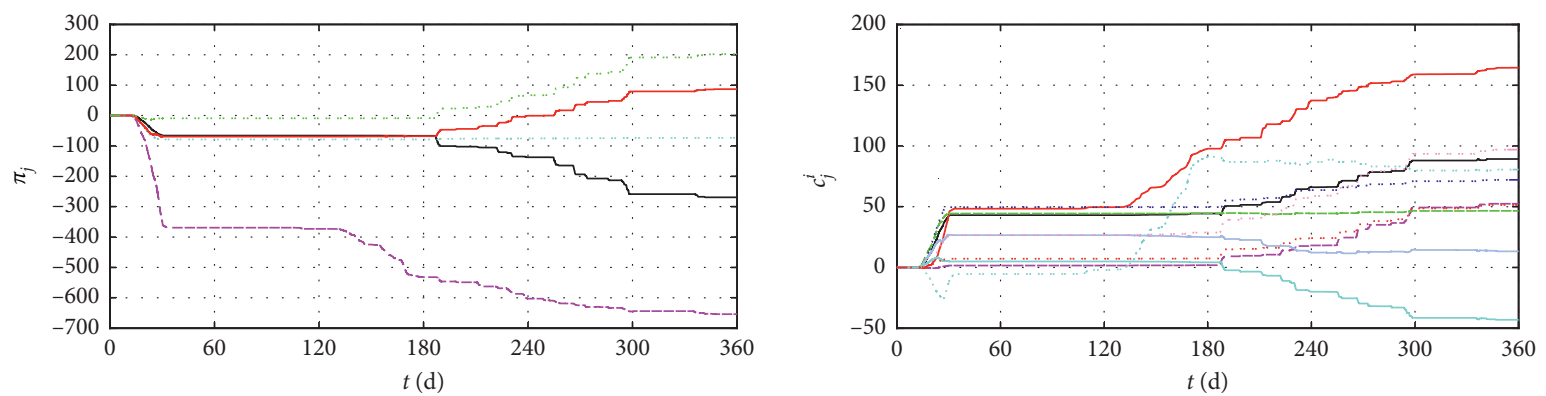

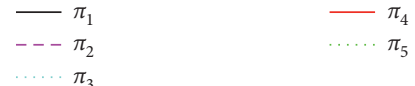

(c)
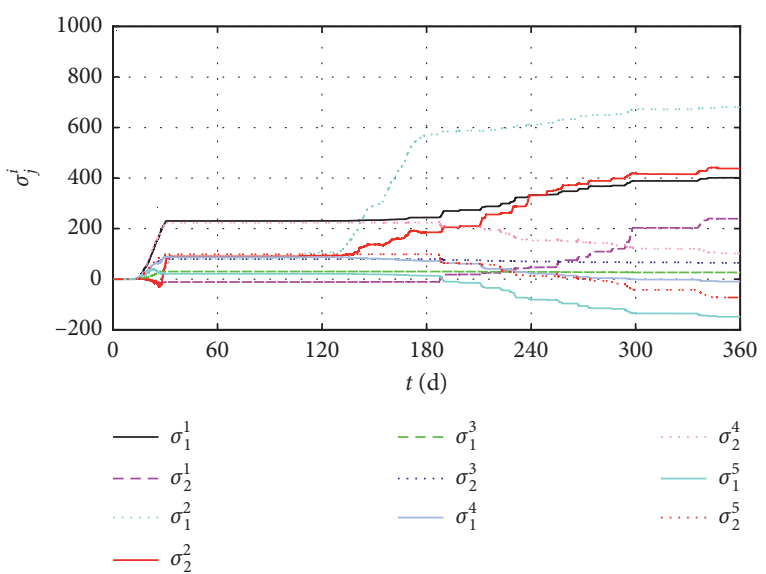

(e) (d)

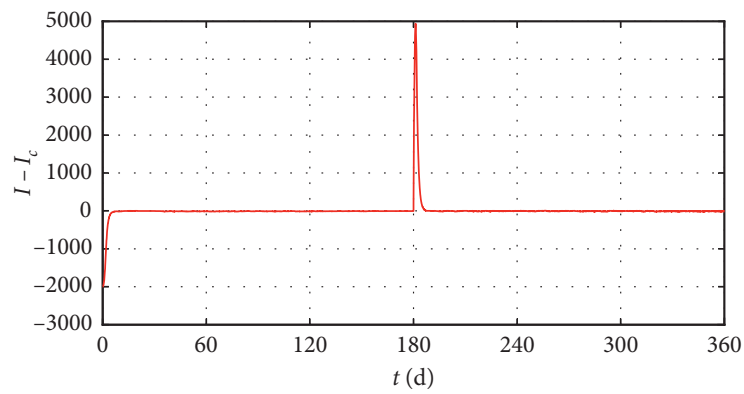

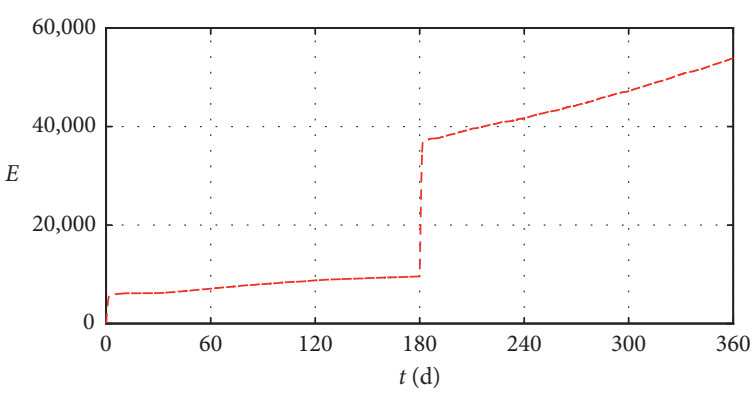

(g)

FiguRe 3: Simulation results under step demand. (a) Customers' demand. (b) Inventory curves. (c) Curves of adaptive parameter $\pi_{j}$. (d) Curves of adaptive parameter $c_{j}^{i}$. (e) Curves of adaptive parameter $\sigma_{j}^{i}$. (f) Error curve of $I-I_{c}$. (g) Curve of $E$. 

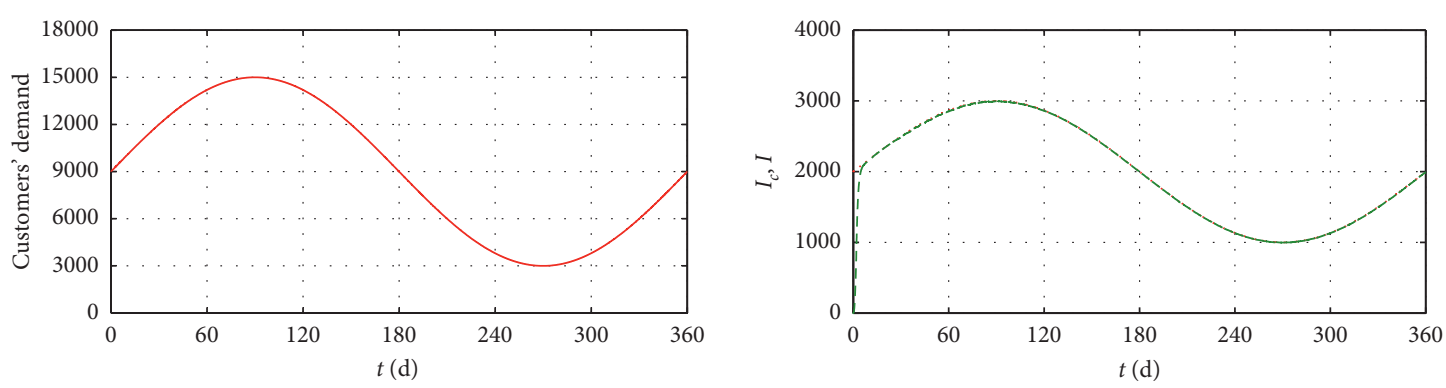

$$
\begin{aligned}
& \ldots . . I_{c} \\
& ---I
\end{aligned}
$$

(a)
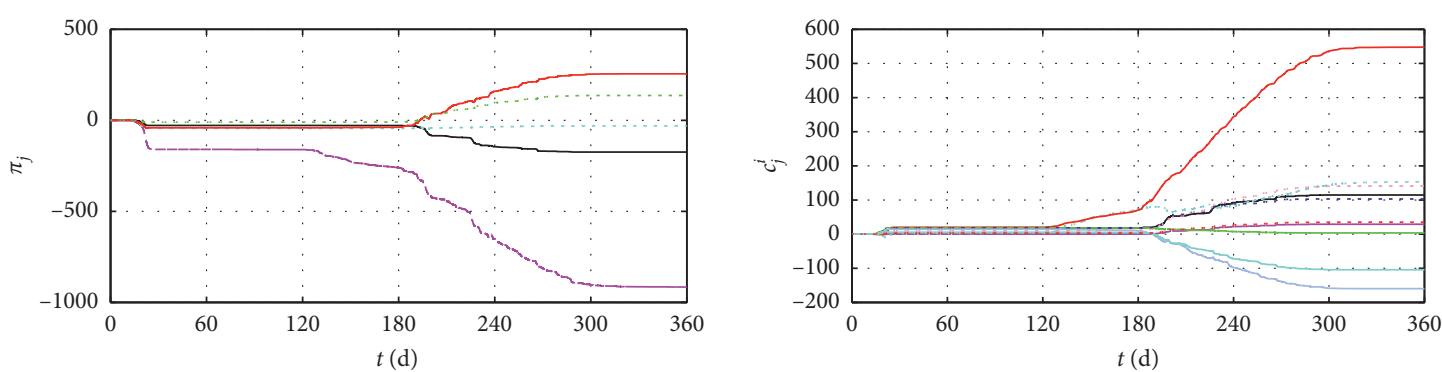

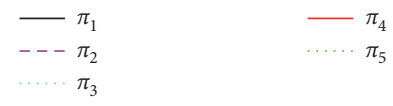

(c)

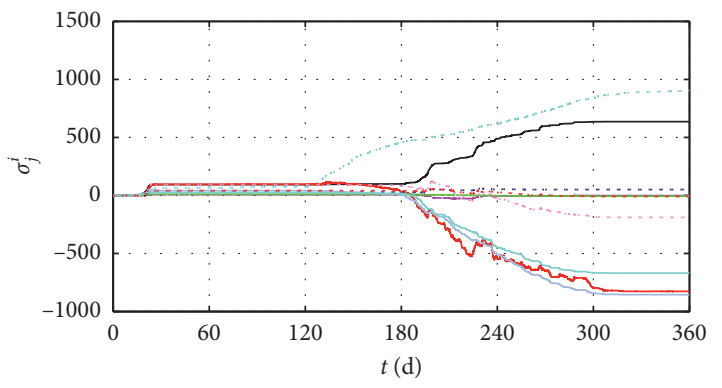

$\begin{array}{rrr}-\sigma_{1}^{1} & --\sigma_{1}^{3} & \cdots \cdots \sigma_{2}^{4} \\ ---\sigma_{2}^{1} & \cdots \cdots \sigma_{2}^{3} & -\sigma_{1}^{5} \\ \cdots \sigma_{1}^{2} & -\sigma_{1}^{4} & \cdots \cdots \sigma_{2}^{5} \\ -\sigma_{2}^{2} & & \end{array}$

(e)

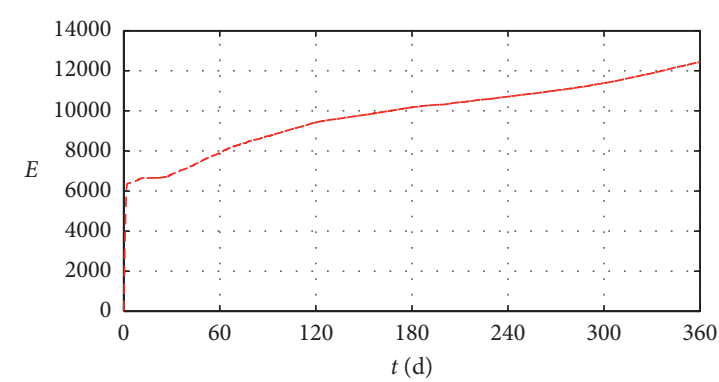

(g) (f)
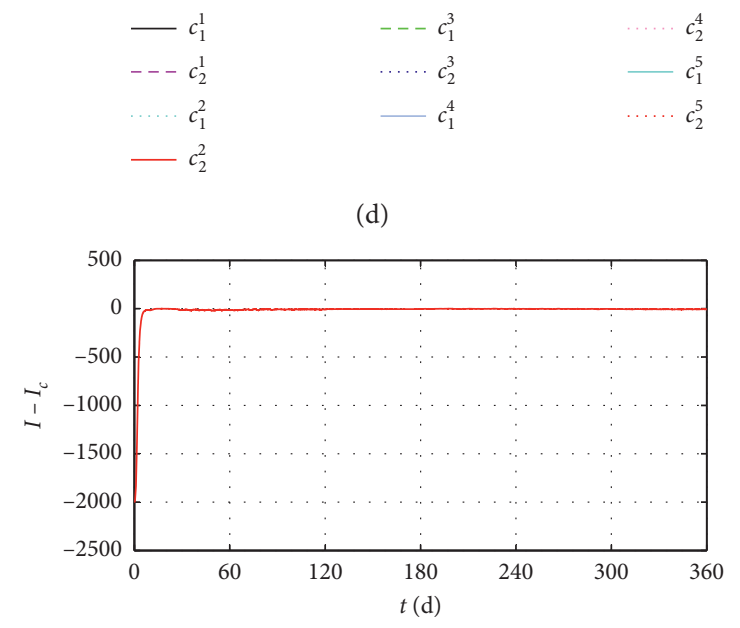

(d)

$\sigma_{2}^{4}$
$\sigma_{1}^{5}$
$\sigma_{2}^{5}$ 


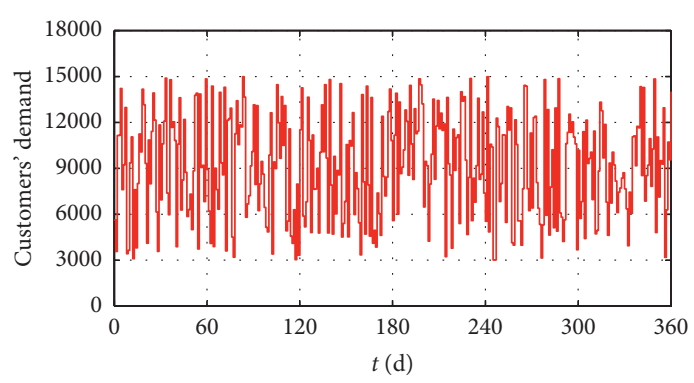

(a)

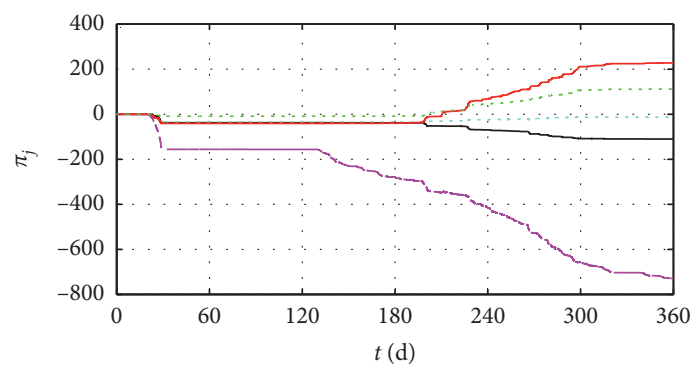

$-\pi_{1}$
$---\pi_{2}$
$\cdots \cdots \pi_{3}$

(c)

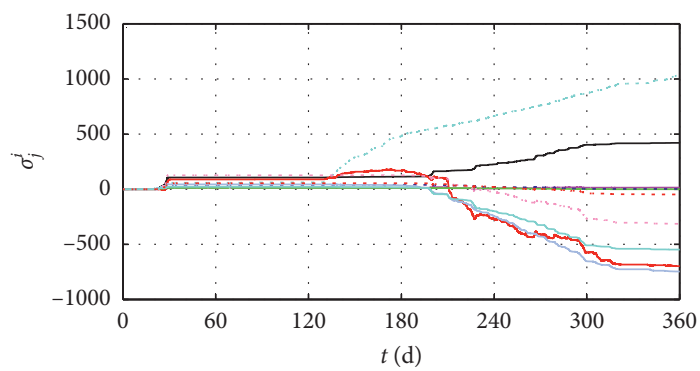

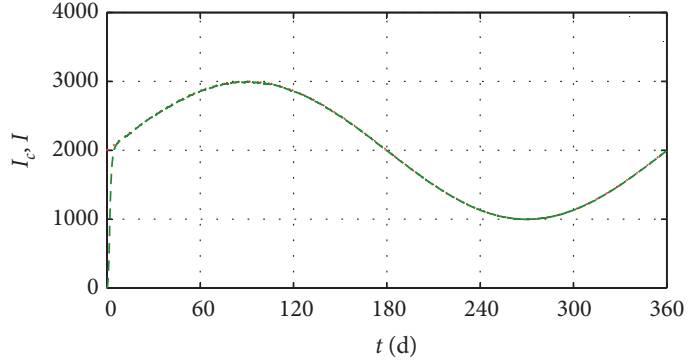

$\ldots \cdot I_{c}$
$---I$

(b)

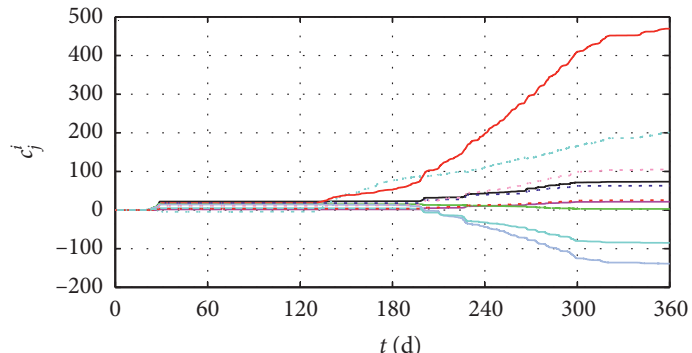

$-c_{1}^{1}$
$---c_{2}^{1}$
$c_{1}^{2}$
$-c_{2}^{2}$

$--c_{1}^{3} \quad \cdots \cdots c_{2}^{4}$

….. $c_{2}^{3}$

$-c_{1}^{4}$

(d)

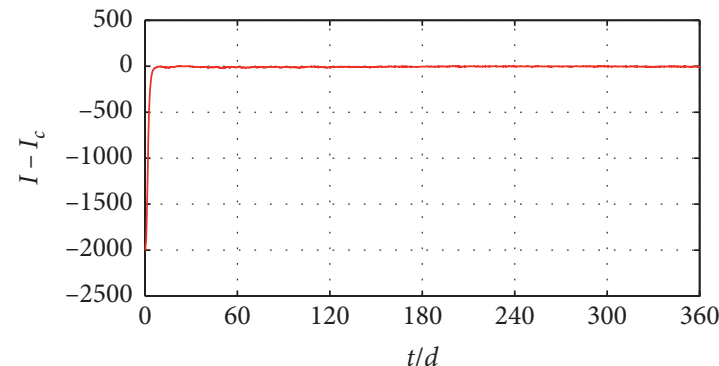

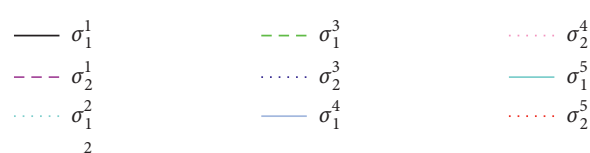

(e) (f)

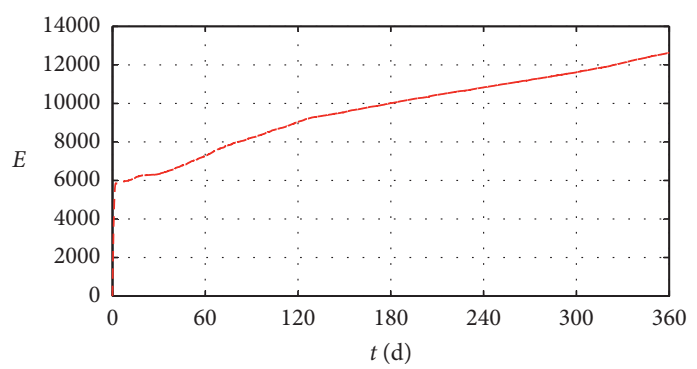

(g)

Figure 5: Simulation results under random demand. (a) Customers' demand. (b) Inventory curves. (c) Curves of adaptive parameter $\pi_{j}$. (d) Curves of adaptive parameter $c_{j}^{i}$. (e) Curves of adaptive parameter $\sigma_{j}^{i}$. (f) Error curve of $I-I_{c}$. (g) Curve of $E$. 


$$
\begin{aligned}
& \dot{V}(t)=e_{1}(t) \dot{e}_{1}(t)+e_{2}(t) \dot{e}_{2}(t)+\frac{\widetilde{E}(t) \dot{\tilde{E}}(t)}{\eta_{1}}+\frac{\tilde{\pi}^{T} \dot{\tilde{\pi}}}{\eta_{2}}+\frac{\tilde{c}^{T} \dot{\tilde{c}}}{\eta_{3}}+\frac{\tilde{\sigma}^{T} \dot{\tilde{\sigma}}}{\eta_{4}} \\
& =e_{1}(t)\left(e_{2}(t)-\tau_{1} e_{1}(t)\right)+e_{2}(t) \\
& \cdot\left(\tilde{\pi}^{T} \bar{\phi}+\tilde{c}^{T} \phi_{c}^{\prime T} \hat{\pi}+\tilde{c}^{T} \phi_{\sigma}^{\prime T} \widehat{\pi}+\varepsilon-\tau_{2} e_{2}(t)-e_{1}(t)\right) \\
& -e_{2}(t) \widehat{E} \operatorname{sgn}\left(e_{2}(t)\right)+\frac{\widetilde{E}(t) \dot{\tilde{E}}(t)}{\eta_{1}}+\frac{\tilde{\pi}^{T} \dot{\tilde{\pi}}}{\eta_{2}}+\frac{\tilde{c}^{T} \dot{\tilde{c}}}{\eta_{3}}+\frac{\tilde{\sigma}^{T} \dot{\tilde{\sigma}}}{\eta_{4}} \\
& =-\tau_{1} e_{1}^{2}(t)-\tau_{2} e_{2}^{2}(t)+\tilde{\pi}^{T}\left(e_{2} \bar{\phi}+\frac{\dot{\tilde{\pi}}}{\eta_{2}}\right)+\tilde{c}^{T}\left(e_{2} \phi_{c}^{\prime} T \widehat{\pi}+\frac{\dot{\tilde{c}}}{\eta_{3}}\right) \\
& +\tilde{\sigma}^{T}\left(e_{2} \phi_{\sigma}^{\prime} \bar{\pi}+\frac{\dot{\tilde{\sigma}}}{\eta_{4}}\right)+\varepsilon e_{2}(t)-\widehat{E}\left|e_{2}(t)\right|+\frac{\widetilde{E}(t) \dot{\tilde{E}}(t)}{\eta_{1}} .
\end{aligned}
$$
have

Because of $\dot{V}\left(e_{1}(t), e_{2}(t), \widetilde{E}(t), \widetilde{\pi}(t), \widetilde{c}(t), \widetilde{\sigma}(t)\right) \leq 0$, we

$$
\begin{aligned}
& \dot{V}\left(e_{1}(t), e_{2}(t), \widetilde{E}(t), \widetilde{\pi}(t), \widetilde{c}(t), \widetilde{\sigma}(t)\right) \\
& \quad \leq V\left(e_{1}(0), e_{2}(0), \widetilde{E}(0), \tilde{\pi}(0), \widetilde{c}(0), \widetilde{\sigma}(0)\right) .
\end{aligned}
$$

Therefore, $e_{1}(t), e_{2}(t), \widetilde{E}(t), \widetilde{\pi}(t), \widetilde{c}(t)$, and $\widetilde{\sigma}(t)$ are bounded, which means that $\bar{\pi}(t), \bar{c}(t)$, and $\bar{\sigma}(t)$ are bounded, then $\Gamma$ is bounded, and $\|\varepsilon\| \leq E$.

Equation (26) can be rewritten as follows:

$$
\begin{aligned}
\dot{V}(t) & =-\tau_{1} e_{1}^{2}(t)-\tau_{2} e_{2}^{2}(t)+\varepsilon e_{2}(t)-E\left|e_{2}(t)\right| \\
& \leq-\tau_{1} e_{1}^{2}(t)-\tau_{2} e_{2}^{2}(t)-(E-|\varepsilon|)\left|e_{2}(t)\right| \\
& \leq-\tau_{1} e_{1}^{2}(t)-\tau_{2} e_{2}^{2}(t) \\
& \leq 0 .
\end{aligned}
$$

Define a function as follows:

$$
\Psi(t)=\tau_{1} e_{1}^{2}(t)+\tau_{2} e_{2}^{2}(t)=-\dot{V}_{1}\left(e_{1}(t), e_{2}(t)\right) .
$$

The integral of equation (29) is as follows

$$
\int_{0}^{t} \Psi(\tau) \mathrm{d} \tau=V_{1}\left(e_{1}(0), e_{2}(0)\right)-V_{1}\left(e_{1}(t), e_{2}(t)\right) .
$$

Because $V_{1}\left(e_{1}(0), e_{2}(0)\right) \quad$ is bounded and $V_{1}\left(e_{1}(t), e_{2}(t)\right)$ is nonincreasing bounded, $\lim _{t \longrightarrow \infty} \int_{0}^{t} \Psi(\tau) \mathrm{d} \tau<\infty$ can be determined. So $\dot{\Psi}(\tau)$ is bounded. In terms of the corollary of the Barbalat lemma [32], $\lim _{t \rightarrow \infty} \Psi(\tau)=0$. That is, $e_{1}(t)$ and $e_{2}(t)$ are asymptotically equal to zero when $t \longrightarrow \infty$. And then $\lim _{t \longrightarrow \infty}\left[I(t)-I_{c}(t)\right]=0$. Therefore, the production-inventory system is asymptotically stable.

\section{Simulation Analysis}

In this section, the production-inventory system of YABAOBAO Down Clothing Company Limited (YBB) is selected as a simulation objective. Set 360 days as the simulation duration, in which the first 180 days represent autumn and winter, and the last 180 days represent spring and summer. Then the sine function $I_{c}=1000 \times \sin (t / 57.3)+2000$ is set as the target inventory of YBB. For sales season of the down garments in autumn and winter, $T_{I}=5$ and $T_{a}=7$; for sales off-season of the down garments in spring and summer, in order to save costs, $T_{I}=7$ and $T_{a}=10$. It is assumed that the demand disturbance of the down garments meets the uniform distribution, that is, $\gamma \sim U(-200,200)$. We will apply the fuzzy neural network composed of five fuzzy rules to estimate the uncertainty $\Omega$ and set the initial values of $\pi, c$, and $\sigma$ are all 0 . Other parameters are set as follows: $\tau_{1}=\tau_{2}=1, \eta_{1}=2.5$, and $\eta_{2}=\eta_{3}=\eta_{4}=50$. To verify the tracking performance of the adaptive inventory controller, the simulation experiments will be carried out under three customers' demands: step function, sine function, and random function.

The simulation results under step demand, sine demand, and random demand are shown in Figures 3-5, respectively.

As shown in Figure 3, the customers' demand is 15000 and the target inventory is 2000 at the beginning of the simulation, and then the actual inventory tracks the target inventory and is almost the same as the target inventory after 6 days. When a step change of the customers' demand from 15000 to 3000 occurs on the 180th day, the actual inventory begins to rise sharply, but the actual inventory tracks down the target inventory on the 187th day. As shown in Figures 4 and 5 , both the actual inventories under the sine demand and the random demand track down the target inventories on the 5th day.

As a result, by adjusting 3 adaptive parameters real-time online, the designed adaptive inventory controller achieves fast and accurate tracking of the target inventory under different customers' demands and uncertain environment and ensures the robust stability of the production-inventory system of YBB.

\section{Conclusions}

Appropriate inventory can effectively enhance the vitality of enterprises. However, inventory level is often subject to the uncertain environment and cannot be consistent with the target inventory. In this paper, the fuzzy neural network has been used to estimate the uncertainty in the inventory control model. Then we designed an adaptive control law consisting of 3 adaptive parameters which can be adjusted real-time online. Based on the designed adaptive control law, an adaptive inventory controller was proposed to implement the actual inventory closely tracking the target inventory. From the simulation results, the proposed adaptive inventory controller can accomplish the following: (1) robustly stable operation of the production-inventory system under uncertain environment, (2) the realtime tracking of dynamic target inventory under different demands, and (3) high tracking speed and accuracy. In this paper, we only studied the adaptive control of single inventory system. Therefore, the adaptive control of multiechelon inventory system can be considered in future research. 


\section{Data Availability}

The simulation data used to support the findings of this study are included within the manuscript. These data are restricted by the Zhejiang China Apparel Network Technology Company Limited in order to protect trade secrets. Data can be available from http://www.efu.com.cn/for researchers who meet the criteria for access to confidential data.

\section{Conflicts of Interest}

The authors declare that they have no conflicts of interest.

\section{Acknowledgments}

The authors are grateful for supports from the Humanity and Social Science Research Foundation of Ministry of Education, China (no. 20YJAZH131), the Shandong Provincial Social Science Plan Foundation, China (no. 19BYSJ13), and the PhD Research Startup Foundation of Linyi University, China (no. 18LUBK01).

\section{References}

[1] T. Avinadav, T. Chernonog, Y. Lahav, and U. Spiegel, "Dynamic pricing and promotion expenditures in an EOQ model of perishable products," Annals of Operations Research, vol. 248, no. 1-2, pp. 75-91, 2017.

[2] S. Tavakoli and A. A. Taleizadeh, "An EOQ model for decaying item with full advanced payment and conditional discount," Annals of Operations Research, vol. 259, no. 3, pp. 1-22, 2017.

[3] R. P. Tripathi, "Establishment of EOQ (Economic Order Quantity) model for spoilage products and power demand under permissible delay in payments," International Journal of Applied and Computational Mathematics, vol. 4, no. 2, p. 55, 2018.

[4] J. Dhandapani and R. Uthayakumar, "Multi-item EOQ model for fresh fruits with preservation technology investment, time-varying holding cost, variable deterioration and shortages," Journal of Control and Decision, vol. 4, no. 2, pp. 70-80, 2016.

[5] A. Chiarini, "An adaptation of the EOQ formula for JIT quasipull system production," Production Planning \& Control, vol. 28, no. 2, pp. 123-130, 2017.

[6] J. J. Liao, K. N. Huang, K. J. Chung, S. D. Lin, P. S. Ting, and H. M. Srivastava, "Retailer's optimal ordering policy in the EOQ model with imperfect-quality items under limited storage capacity and permissible delay," Mathematical Methods in the Applied Sciences, vol. 41, no. 17, pp. 7624-7640, 2018.

[7] D. Castellano, E. Gebennini, A. Grassi, T. Murino, and B. Rimini, "Stochastic modeling of a single-vendor singlebuyer supply chain with $(s, S)$-inventory policy," IFACPapersOnLine, vol. 51, no. 11, pp. 974-979, 2018.

[8] O. J. Boxma, D. Perry, and W. Stadje, "The $(S-1, S)$ inventory model and its counterparts in queueing theory," Operations Research Letters, vol. 47, no. 6, pp. 483-488, 2019.

[9] R. Qiu, M. Sun, and Y. F. Lim, "Optimizing $(s, S)$ policies for multi-period inventory models with demand distribution uncertainty: robust dynamic programing approaches,"
European Journal of Operational Research, vol. 261, no. 3, pp. 880-892, 2017.

[10] R. Singh, A. Singh, and A. Agarwal, "Modification and analysis of APIOBPCS model using inventory control best practices for FMCG sector," International Journal of Basic and Applied Sciences, vol. 4, no. 3, pp. 108-117, 2015.

[11] W. F. Xie and J. H. Ma, "Optimization of a vendor managed inventory supply chain based on complex fuzzy control theory," WSEAS Transactions on Systems, vol. 13, pp. 429-439, 2014.

[12] A. Hosseini and Y. Z. Mehrjerdi, "The bullwhip effect on the VMI-supply chain management via system dynamics approach: the supply chain with two suppliers and one retail channel," International Journal of Supply and Operations Management, vol. 3, no. 2, pp. 1301-1317, 2016.

[13] T. Hosoda and S. M. Disney, "On the replenishment policy when the market demand information is lagged," International Journal of Production Economics, vol. 135, no. 1, pp. 458-467, 2012.

[14] S. M. Disney and D. R. Towill, "On the bullwhip and inventory variance produced by an ordering policy," Omega, vol. 31, no. 3, pp. 157-167, 2003.

[15] J. Wikner, M. M. Naim, and M. Rudberg, "Exploiting the order book for mass customized manufacturing control systems with capacity limitations," IEEE Transactions on Engineering Management, vol. 54, no. 1, pp. 145-155, 2007.

[16] X. Zhan, Q. Zhang, and W. Xie, "Dynamic performance and stability research of VMI-APIOBPCS in apparel industry based on control theory," International Journal of Enterprise Information Systems, vol. 14, no. 2, pp. 56-76, 2018.

[17] K. Subramanian, J. B. Rawlings, and C. T. Maravelias, "Economic model predictive control for inventory management in supply chains," Computers \& Chemical Engineering, vol. 64, pp. 71-80, 2014.

[18] M. Y. Cui, H. Z. Liu, and W. Liu, "Extended state observerbased adaptive control for a class of nonlinear system with uncertainties," Control and Intelligent Systems, vol. 45, no. 3, pp. 132-141, 2017.

[19] W. Deng, J. Yao, and D. Ma, “Time-varying input delay compensation for nonlinear systems with additive disturbance: an output feedback approach," International Journal of Robust and Nonlinear Control, vol. 28, no. 1, pp. 31-52, 2017.

[20] E. Aggelogiannaki, P. Doganis, and H. Sarimveis, "An adaptive model predictive control configuration for production-inventory systems," International Journal of Production Economics, vol. 114, no. 1, pp. 165-178, 2008.

[21] A. Foul, L. Tadj, and R. Hedjar, "Adaptive control of inventory systems with unknown deterioration rate," Journal of King Saud University-Science, vol. 24, no. 3, pp. 215-220, 2012.

[22] K. Li, K. H. Chan, B. E. Ydstie, and R. Bindlish, "Passivitybased adaptive inventory control," Journal of Process Control, vol. 20, no. 10, pp. 1126-1132, 2010.

[23] A. Mandel, "Models and algorithms of inventory control in case of uncertainty," IFAC Proceedings Volumes, vol. 42, no. 4, pp. 223-228, 2009.

[24] P. Aengchuan and B. Phruksaphanrat, "Comparison of fuzzy inference system (FIS), FIS with artificial neural networks $($ FIS + ANN) and FIS with adaptive neuro-fuzzy inference system (FIS + ANFIS) for inventory control," Journal of Intelligent Manufacturing, vol. 29, no. 4, pp. 905-923, 2018.

[25] V. N. Azarskov, L. S. Zhiteckii, K. Y. Solovchuk, O. A. Sushchenko, and R. O. Lupoi, "Inventory control for a manufacturing system under uncertainty: adaptive approach," IFAC-PapersOnLine, vol. 50, no. 1, pp. 10154-10159, 2017. 
[26] P. Vladimir and J. P. Kenne, "Adaptive control of manufacturing systems with incomplete information about demand and inventory," IFAC-PapersOnLine, vol. 50, no. 1, pp. 15598-15603, 2017.

[27] S. K. Paul, A. Azeem, and A. K. Ghosh, "Application of adaptive neuro-fuzzy inference system and artificial neural network in inventory level forecasting," International Journal of Business Information Systems, vol. 18, no. 3, pp. 268-284, 2015.

[28] D. Fu, C. Ionescu, E.-H. Aghezzaf, and R. De Keyser, "Quantifying and mitigating the bullwhip effect in a benchmark supply chain system by an extended prediction selfadaptive control ordering policy," Computers \& Industrial Engineering, vol. 81, pp. 46-57, 2015.

[29] L. X. Wang, "Stable and optimal fuzzy control of linear systems," IEEE Transactions on Fuzzy Systems, vol. 6, no. 1, pp. 137-143, 1998.

[30] Q. Wang and C. Sun, "Adaptive consensus of multiagent systems with unknown high-frequency gain signs under directed graphs," IEEE Transactions on Systems, Man, and Cybernetics: Systems, vol. 50, no. 6, pp. 2181-2186, 2020.

[31] Q. Wang, H. E. Psillakis, and C. Sun, "Cooperative control of multiple agents with unknown high-frequency gain signs under unbalanced and switching topologies," IEEE Transactions on Automatic Control, vol. 64, no. 6, pp. 2495-2501, 2019.

[32] Z. Wu, Y. Xia, and X. Xie, "Stochastic Barbalat's lemma and its applications," IEEE Transactions on Automatic Control, vol. 57, no. 6, pp. 1537-1543, 2012. 